\title{
Report of the 2021 Primary Ciliary Dyskinesia Foundation annual meeting
}

\author{
Thomas G. Saba ${ }^{\mathrm{a}, *}$ and Steven L. Brody ${ }^{\mathrm{b}}$ \\ ${ }^{a}$ Department of Pediatrics, University of Michigan, Ann Arbor, MI, USA \\ ${ }^{\mathrm{b}}$ Department of Medicine, Washington University in Saint Louis, Saint Louis, MO, USA
}

\begin{abstract}
In August 2021, the Primary Ciliary Dyskinesia (PCD) Foundation hosted a 2-day international virtual conference designed to share discoveries in PCD genetics, cilia biology, and clinical research and care. The conference was organized around the theme of building a community for clinical research. This article provides a report of the proceedings.
\end{abstract}

Keywords: Primary ciliary dyskinesia, cilia, genetics, rare diseases

\section{Introduction}

Primary ciliary dyskinesia (PCD) is a rare genetic disease caused by impaired motile cilia structure or function. Patients often have respiratory distress at birth, body laterality defects, congenital heart disease, a lifetime of chronic upper and lower respiratory tract infections, and infertility. The first genetic cause for PCD was identified less than 20 years ago. The pathophysiology, genetics, and disease-specific treatments, therefore, are still at the early stages of discovery. The Primary Ciliary Dyskinesia Foundation (PCDF), founded in 2001 by the steadfast executive director, Michele Manion, has built a growing network of 30 PCDF Clinical and Research Centers in North America in an effort to provide specialized PCD care. An annual or semiannual meeting has provided a platform to share new discoveries and guidelines between centers and investigators worldwide. In 2021, the PCDF held a virtual conference over two days for a diverse group of clinicians, clinical investigators, scientists, patients, and families to exchange current knowledge and share a vision of future scientific discovery and clinical care. The conference successfully engaged over 330 registrants from 29 countries. In light of the approaching launch of the PCDF patient registry, the theme of the conference was: "Building a Community for Clinical Research".

\section{PCD Genetics: Innovations and discoveries}

With expanded availability of relatively inexpensive commercial genetic testing for PCD diagnosis, a thorough understanding of the molecular biology and genetics of PCD is critical. Amelia Shoemark, $\mathrm{PhD}$, from the University of Dundee, presented a detailed classification of human mutations affecting

\footnotetext{
${ }^{*}$ Corresponding author: Thomas Saba, Department of Pediatrics, University of Michigan, 1500 E. Medical Center Drive, Ann Arbor, MI 48109, USA. Tel.: +1 734764 4124; Fax: +1 734936 7635; E-mail: tsaba@med.umich.edu.
} 
ciliary structure and motility [1]. She demonstrated how a combination of transmission electron microscopy, high speed video microscopy, immunofluorescence, and genetic sequencing are needed to study the subtle, complex and variable impact of mutant proteins on ciliary structure and function. William Hannah, MD, from the University of North Carolina, Chapel Hill, sought to estimate prevalence and ethnic heterogeneity of PCD based on the allele frequency of genetic variants in the Genome Aggregation Database (gnomAD), an international resource for aggregating and harmonizing sequencing data [2]. Using strict criteria for analysis of variants (including variants of unknown significance) for 29 PCD-associated genes, Hannah estimates PCD prevalence to be about twice the traditionally quoted estimate of 1 in 20,000. Exploratory work uncovered a difference in genetic variants among different ethnic populations; he pointed out that a higher frequency of PCD may be present and under diagnosed in African American populations. Next, in an attempt to correlate genetic mutations with clinical outcomes, BreAnna Kinghorn, MD, from the University of Washington, described findings derived from a longitudinal database from the Genetic Disorders of Mucociliary Clearance Consortium (GDMCC) [3]. Lung function and chest CT scores were correlated with ciliary ultrastructural and genetic defects as classified by Shoemark. Kinghorn reported that patients with mutations leading to inner dynein arm defects with microtubule disorganization (IDA-MTD) (CCDC39 or CCDC40 mutations) had worse lung function, more rapid lung function decline, and worse chest CT imaging scores compared to the more common outer dynein arm defects (e.g., DNAH5, DNAH11, DNAI1 genes). Interestingly, forced expiratory volume in 1 second (FEV1) among those subjects with IDA-MTD defects was lower than that of age-matched children with cystic fibrosis displaying minimal CFTR function. Chest imaging was assessed using a novel scoring system adapted for PCD by incorporating atelectasis, bronchiectasis, bronchial wall thickening, and mucus plugging. This CT scoring approach may be considered for future multicenter validation. Susan Dutcher, $\mathrm{PhD}$, from Washington University in St. Louis, illuminated the CCDC39-CCDC40 conundrum by describing ground-breaking findings in cilia imaging technology using high resolution cryo-electron microscopy and proteomics to resolve ciliary proteins at amino acid levels [4]. Reconstructed images of the cilium showed how $C C D C 39$ is entwinned with its partner, $C C D C 40$, and interacts with critical structural and regulatory proteins. Complementary proteomic data from human CCDC39 mutant cilia suggested extensive loss of structural and regulatory proteins throughout the ciliary axoneme, perhaps accounting for the severe phenotype.

\section{Clinical care and research}

In an effort to extend expert PCD care to patients in Latin American, the organizers invited Ana Maria Herrara Gana, MD, from Universidad de los Andes in Santiago, Chile, to share her experience building international partnerships to develop a successful PCD diagnostic and treatment center. Major scientific contributions from GDMCC studies over the last 20 years were summarized by Adam Shapiro, MD, from McGill University. In an entertaining "greatest hits" format, Shapiro recapped how the GDMCC investigators' establishment of a longitudinal cohort of PCD patients contributed to the discovery of PCD gene mutations, diagnostic criteria, validation of nasal nitric oxide testing, and the study of genotype-phenotype correlations. Next, challenges encountered when launching the first North American-based therapeutic studies for PCD were summarized by Parion Science's Vice President of Drug Development, Karl Donn, PhD. Donn described a steep learning curve for both PCD centers and patients, as both parties have limited experience participating in clinical trials. The paucity of PCD therapeutic trials has been of concern to the PCDF. To address this gap, Michael O'Connor, MD, from Vanderbilt University, introduced the PCDF registry, constructed with the goal of creating an ecosystem of data and clinical information to serve as a platform for large-scale clinical studies and 
improved clinical care of individuals with PCD. O'Connor described the registry's early rollout and plan to bring all North American centers online.

\section{Scientific abstracts}

Several investigators used the virtual format to share posters and oral presentations describing research in PCD. Ongoing clinical studies performed in the GDMCC network explore diverse topics including PCD treatments patterns, the use of artificial intelligence to identify cases of PCD from electronic medical records, and relationships between genotype, ciliary ultrastructure and clinical phenotypes-including neonatal respiratory distress, laterality defects, oto-sinus manifestations, and chest CT findings. Other groups shared how the Covid-19 pandemic impacted PCD patient mental health as well as infection prevention and control measures in PCD clinics. Two biotechnology companies, Re-code and Translate Bio, each reported preliminary data regarding the promising use of gene specific, mRNA-based therapies to rescue ciliary function in DNAII deficient airway epithelial cells. Several abstracts described findings that helped to expand the understanding of ciliary structure and function, and phenotypic impact of certain gene mutations. Topics included the expression of cytoplasmic proteins which compose the dynein axonemal assembly complex, a murine model of mild human disease due to a DNAAF5 mutation, and a correlation of a unique mutant CCDC114 allele with a mild human PCD phenotype. Finally, the unexpected extent of bronchiectasis among a population of Puerto Rican PCD patients with RSPH4A mutations was described.

\section{Lessons from clinical cases}

In what has become a tradition at the PCDF meetings, unique clinical cases were presented, including a mild case of PCD in an elderly person secondary to RSPH1 mutations, a child with a hemizygous $R P G R$ mutation with improved bronchiectasis after initiation of treatment, and two cases of primary immunodeficiency identified in patients thought initially to have PCD. The cases drew interest from conference participants with similar experiences and triggered the development of a working group of clinicians interested in exploring primary immunodeficiencies among patients initially thought to have PCD.

\section{An opportunity for patients and families}

The risk for patient-to-patient infection transmission generally prohibits patients from attending conferences. However, the virtual platform offered a unique opportunity for patients and families to attend all aspects of the conference. Each day ended with an hour session for patients and families to ask PCD experts questions. Live interactions with PCD clinicians helped to consolidate a PCDF mission of patient- and family-centered, collaborative medical care.

\section{Growing the mission of the PCDF}

The meeting presenters were largely junior investigators and clinicians, signifying a promising future for PCD research. In summary, meeting highlights included: welcoming new members of the PCD community, learning about exciting new discoveries in ciliary biology, and establishing a shared commitment to building a national patient care and research network, as well as extending international partnerships. 


\section{References}

[1] J.S. Lucas, S.D. Davis, H. Omran and A. Shoemark, Primary ciliary dyskinesia in the genomics age, Lancet Respir Med 8 (2020), 202-216.

[2] K.C. de Andrade, M.N. Frone, T. Wegman-Ostrosky, P.P. Khincha, J. Kim, A. Amadou, K.M. Santiago, F.P. Fortes, N. Lemonnier, L. Mirabello, D.R. Stewart, P. Hainaut, L.P. Kowalski, S.A. Savage and M.I. Achatz, Variable population prevalence estimates of germline TP53 variants: A gnomAD-based analysis, Hum Mutat 40 (2019), 97-105.

[3] S.D. Davis, M. Rosenfeld, H.S. Lee, T.W. Ferkol, S.D. Sagel, S.D. Dell, C. Milla, J.E. Pittman, A.J. Shapiro, K.M. Sullivan, K.R. Nykamp, J.P. Krischer, M.A. Zariwala, M.R. Knowles and M.W. Leigh, Primary Ciliary Dyskinesia: Longitudinal Study of Lung Disease by Ultrastructure Defect and Genotype, Am J Respir Crit Care Med 199 (2019), 190-198.

[4] M. Ma, M. Stoyanova, G. Rademacher, S.K. Dutcher, A. Brown and R. Zhang, Structure of the Decorated Ciliary Doublet Microtubule, Cell 179 (2019), 909-922, e912. 